\title{
Performance of Wideband CDMA Systems with Complex Spreading and Imperfect Channel Estimation
}

\author{
Jiangzhou Wang, Senior Member, IEEE and Jun Chen
}

\begin{abstract}
The coherent RAKE reception of wideband code division multiple access (W-CDMA) signals with complex spreading is considered in this paper. A general multipath-fading channel model is assumed. A dedicated pilot channel, which is separate from data channels, is used for the purpose of channel estimation. Based on a digital implementation, the coherent demodulation scheme is presented. Pilot channel estimation error, due to multiple access and multipath interference, is studied. System performance is evaluated by means of bit error rate (BER). The analysis shows that the error of channel estimation significantly degrades system performance and can be effectively suppressed by low pass filters (LPFs). A discussion on the envelope variation of complex spread signals is also included, which illustrates that the complex spread signal has more stable envelope than the dualchannel spread signal. The power ratio of pilot to data channels should be chosen in the range of 0.2 to 0.4 (or -7 to $-4 \mathrm{~dB}$ ), in order to achieve maximum system capacity.
\end{abstract}

Index Terms-Channel estimation, complex spreading, RAKE reception, wideband CDMA.

\section{INTRODUCTION}

$\mathbf{T}$ O PROVIDE higher data rates for end users, as well as to accommodate more users over wireless channels in the future mobile communication systems, wideband direct sequence (DS) code division multiple access (W-CDMA) has become the focus of current research interests. Two of the important features of wideband CDMA systems are the use of complex spreading and user-dedicated pilot channel [1]-[4]. In the W-CDMA standard of the Third Generation Partnership Project (3GPP), a time-multiplexed pilot is used in the downlink, whereas an in-phase/quadrature (I/Q) code-multiplexed pilot is used in the uplink [4]. In this paper, an I/Q code-multiplexed pilot is adopted. However, the results obtained can be applied to a time-multiplexed pilot as well. In addition, the power ratio of pilot to data channels has been evaluated in order to achieve maximum system capacity.

Complex spreading is a term used in contrast to dual-channel spreading [5]. It can be implemented either by a complex valued sequence [6], [7], e.g., polyphase sequence, or by two binary sequences. It is claimed that complex spreading can reduce the peak-to-average power ratio of modulated signals, thus improving RF power amplifier efficiency, but no explicit

\footnotetext{
Manuscript received June 1, 2000; revised October 1, 2000.

The authors are with the Department of Electrical and Electronic Engineering, University of Hong Kong, Hong Kong (e-mail: jwang@eee.hku.hk; junchen@eee.hku.hk).

Publisher Item Identifier S 0733-8716(01)01178-7.
}

results have been given [3]. This declaration is studied in this paper, and some useful results are presented.

Coherent reception outperforms noncoherent by $3 \mathrm{~dB}$ in additive white Gaussian noise (AWGN) channels. In multipath fading channels, a coherent RAKE receiver with maximum ratio combining (MRC) has the optimal performance. Eng and Milstein [8], [9] and Efthymoglou et al. [10] presented the performance of coherent DS-CDMA in Nakagami-fading channels. Coherent reception requires the knowledge of channel characteristics, which are time-varying in fading environments. A conventional and effective method to accomplish this task is to use a separate pilot channel or insert pilot symbols in data symbols. Ling [11] described the optimal estimators for pilot symbols in a pilot channel scheme. The relative mean square error and optimal power allocation between data and pilot signals were also studied. Higuchi et al. [12] and Abeta et al. [13] presented a weighted multislot averaging (WMSA) channel estimation filter for time-multiplexed pilot channel. Choi [14] proposed an adaptive method to estimate channel parameters by jointly utilizing pilot and data channels. However, the analytical expression of the channel estimation error has been insufficient. In this paper, the channel estimation error is analyzed in a multiple access and multipath environment, which leads to a better understanding of the nature of this estimation error and possible methods to reduce the error. The system performance degradation due to the estimation error is also investigated.

This paper is organized as follows. In Section II, the transmitter model, channel model, and receiver structure are presented. The analysis of channel estimation error and system performance is given in Section III, while numerical results are discussed in Section IV. A study on the envelope variation is provided in Section V. Finally, some conclusions are drawn in Section VI.

\section{SYSTEM MODELS}

\section{A. Transmitter Model}

In the uplink of wideband CDMA systems, where an I/Q code-multiplexed pilot is utilized, spreading consists of two operations. The first is channelization operation, in which pilot and data symbols on I- and Q-branches are independently multiplied with an orthogonal variable spreading factor (OVSF) code and transformed into a number of chips. The second operation is scrambling, where the resultant signals are further multiplied by a complex valued scrambling code. This spreading scheme is called complex spreading, as illustrated in Fig. 1. 


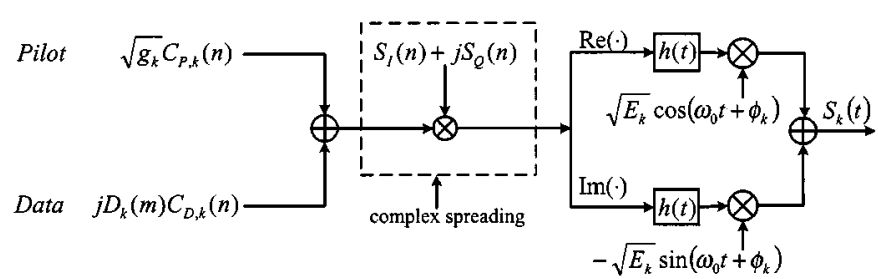

Fig. 1. Transmitter block diagram of the $k$ th user.

The transmitted signal of the $k$ th user can be written as

$$
\begin{aligned}
S_{k}(t)= & \sqrt{E_{D, k}} \sum_{n=-\infty}^{\infty} \\
& \cdot\left[\sqrt{g_{k}} C_{P, k}(n) S_{I}(n)-D_{k}(m) C_{D, k}(n) S_{Q}(n)\right] \\
& \cdot h\left(t-n T_{C}\right) \cos \left(\omega_{0} t+\phi_{k}\right) \\
& -\sqrt{E_{D, k}} \sum_{n=-\infty}^{\infty} \\
& \cdot\left[\sqrt{g_{k}} C_{P, k}(n) S_{Q}(n)+D_{k}(m) C_{D, k}(n) S_{I}(n)\right] \\
& \cdot h\left(t-n T_{c}\right) \sin \left(\omega_{0} t+\phi_{k}\right)
\end{aligned}
$$

where

$\begin{array}{ll}E_{D, k} & \begin{array}{l}\text { chip energy of data channel; } \\ g_{k}\end{array} \\ \begin{array}{l}\text { power ratio of pilot channel to the } \\ D_{k}(m)\end{array} & \begin{array}{l}\text { data channel; } \\ \text { data symbols for the } k \text { th user; }\end{array} \\ & \text { integer part of } n / N, \text { i.e., } m \\ & {[n / N] ;}\end{array}$

Assuming that $\int_{-(A-1) T c / 2}^{(A+1) T c / 2} h^{2}(t) d t=1$, the power of the transmitted signal is

$$
P_{k}=\left(1+g_{k}\right) E_{D, k} / T_{c}=E_{k} / T_{c}
$$

where $E_{k}$ is the chip energy for both data channel and dedicate pilot channel of the $k$ th user.

\section{B. Channel Model and Receiver Structure}

The complex lowpass equivalent impulse response of a multipath fading channel can be written as

$$
h_{k}(t)=\sum_{l=0}^{L-1} \alpha_{k, l}(t) \delta\left[t-\tau_{k, l}(t)\right] e^{j \theta_{k, l}(t)}
$$

where $L(L \geq 1)$ is the number of resolvable propagation paths. For the sake of simple notation, it is assumed that all

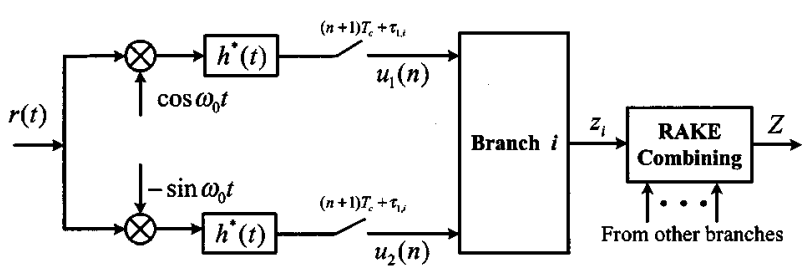

Fig. 2. RAKE receiver block diagram.

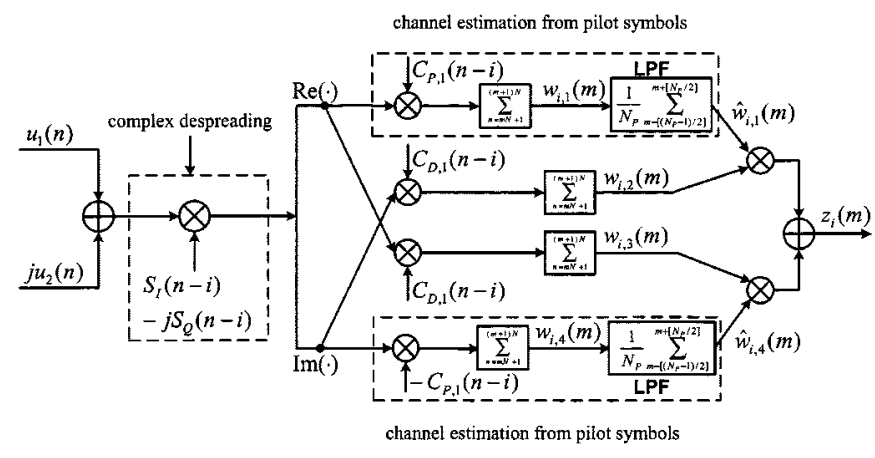

Fig. 3. Detailed demodulation block diagram of the $i$ th branch.

users have the same number of multipaths. $\alpha_{k, l}(t) e^{j \theta_{k, l}(t)}$ and $\tau_{k, l}(t)$ are the complex fading factor and propagation delay of the $l$ th path of the $k$ th user, respectively. Note that $\alpha_{k, l}(t)$ can be Rayleigh-, Rician- or Nakagami-distributed, depending on a specific channel model. All random variables in (3) are assumed independent for $k$ and $l$. Assuming that there are $K$ active users in the system, the received signal is given by

$$
r(t)=\eta(t)+\sum_{k=1}^{K} \sum_{l=0}^{L-1} \alpha_{k, l}(t) S_{k}\left[t-\tau_{k, l}(t)\right] e^{j \theta_{k, l}(t)}
$$

where $\eta(t)$ is the background AWGN with double-side power spectrum density $\eta_{\mathrm{O}} / 2$.

In order to mitigate multipath effect, a RAKE receiver with pilot symbol aided coherent demodulation and maximum ratio combining (MRC) is employed. The RAKE receiver structure is shown in Fig. 2, where the number of branches is less or equal to the number of resolvable paths. The received signal is multiplied by local carriers and passed through the pulse-matching filters and then sampled once per chip duration. The samples are fed to each branch of the RAKE receiver, where the pilot and data symbols are separately demodulated, and pilot symbols are used to eliminate the phase error of different path and achieve MRC. Assuming that the $i$ th path delay $\tau_{1, i}$ can be accurately estimated for the reference user $(k=1)$, each path that corresponds to a RAKE branch gives an output component. The outputs of all branches are added together to form the decision statistic. The detailed demodulation block diagram of the $i$ th branch is shown in Fig. 3.

\section{Channel Estimation Error And Bit Error Rate}

The two sampled outputs in Fig. 2 are given by

$u_{1}(n)=\int_{n T_{c}+\tau_{1, i}}^{(n+1) T_{c}+\tau_{1, i}} r(t) \cos \left(\omega_{0} t\right) h^{*}\left(t-n T_{c}-\tau_{1, i}\right) d t$ 
$u_{2}(n)=-\int_{n T_{c}+\tau_{1, i}}^{(n+1) T_{c}+\tau_{1, i}} r(t) \sin \left(\omega_{0} t\right) h^{*}\left(t-n T_{c}-\tau_{1, i}\right) d t$

where $h^{*}(t)$ is the complex conjugate of $h(t)$. For simple notation, $n T_{c}$ is replaced by $n$. Neglecting the high-frequency component, the two samples can be further written as

$$
\begin{aligned}
u_{1}(n)= & \sum_{k=1}^{K} \sum_{l=0}^{L-1} \beta_{k, l}^{(c)}(n) \\
& \cdot\left(\sqrt{g_{k}} \cdot S_{I}(n-l) C_{P, k}(n-l)\right. \\
& \left.\quad-D_{k}\left(m_{k}\right) \cdot S_{Q}(n-l) C_{D, k}(n-l)\right) \\
& -\sum_{k=1}^{K} \sum_{l=0}^{L-1} \beta_{k, l}^{(s)}(n) \\
& \cdot\left(\sqrt{g_{k}} \cdot S_{Q}(n-l) C_{P, k}(n-l)\right. \\
& \left.+D_{k}\left(m_{k}\right) \cdot S_{I}(n-l) C_{D, k}(n-l)\right)+\eta_{1}(n) \\
u_{2}(n)= & \sum_{k=1}^{K} \sum_{l=0}^{L-1} \beta_{k, l}^{(s)}(n) \\
& \cdot\left(\sqrt{g_{k}} \cdot S_{I}(n-l) C_{P, k}(n-l)\right. \\
& \left.\quad-D_{k}\left(m_{k}\right) \cdot S_{Q}(n-l) C_{D, k}(n-l)\right) \\
& +\sum_{k=1}^{K} \sum_{l=0}^{L-1} \beta_{k, l}^{(c)}(n) \\
& \cdot\left(\sqrt{g_{k}} \cdot S_{Q}(n-l) C_{P, k}(n-l)\right. \\
& \left.+D_{k}\left(m_{k}\right) \cdot S_{I}(n-l) C_{D, k}(n-l)\right)+\eta_{2}(n)
\end{aligned}
$$

where $m_{k}=\left[\left(n-n_{k}\right) / N\right]$ with $n_{k}=\left[\tau_{k, l}\left(n T_{c}\right) / T_{c}\right]$. Since data of interfering users have no effect on variance of multiple access interference, $m_{k}$ is replaced by $m$ for simple notation. $\eta_{1}(n)$ and $\eta_{2}(n)$ are the sampled noise components, and

$$
\begin{aligned}
& \beta_{k, l}^{(c)}(n)=\frac{1}{2} \sqrt{E_{D, k}} \alpha_{k, l}(n) R\left[\bar{\tau}_{k, l}(n)\right] \cos \left[\varphi_{k, l}(n)\right] \\
& \beta_{k, l}^{(s)}(n)=\frac{1}{2} \sqrt{E_{D, k}} \alpha_{k, l}(n) R\left[\bar{\tau}_{k, l}(n)\right] \sin \left[\varphi_{k, l}(n)\right]
\end{aligned}
$$

where $\varphi_{k, l}(n)=\phi_{k}+\theta_{k, l}\left(n T_{c}\right)-\omega_{0} \tau_{k, l}\left(n T_{c}\right)$ is the carrier phase offset; $\bar{\tau}_{k, l}(n)$ is the chip-timing error given by $\bar{\tau}_{k, l}(n)=$ $\tau_{k, l}\left(n T_{c}\right)-\tau_{1, i}-\left[\left(\tau_{k, l}\left(n T_{c}\right)-\tau_{1, i}\right) / T_{c}\right] T_{c}$, and $\left|\bar{\tau}_{k, l}(n)\right| \leq$ $T_{c}$; and $R(\bar{\tau})$ is the effect of timing error $\bar{\tau}$ on the sampled outputs. When the impulse response of the shaping filter is limited to $T_{c}$, it has been proved in the Appendix that

$$
R(\bar{\tau})=\int_{-\infty}^{\infty}|H(f)|^{2} \cos (2 \pi f \bar{\tau}) d f
$$

where $H(f)$ is the frequency response of the pulse shaping filter.

Assuming that acquisition and synchronization have been accomplished for the reference user $(k=1)$, the local spreading codes in the $i$ th branch are aligned with the $i$ th path of the received signal. After descrambling by a complex scrambling code, the real and imaginary parts of the resultant signal are multiplied by separate channel codes, respectively, and then accumulated in one symbol period with symbol synchronization $(N$ chips). The four outputs of the $i$ th branch are obtained at four sub-branches after this correlation, as show in Fig. 3, which are given by

$$
\begin{aligned}
w_{i, 1}(m)= & \sum_{n=m N+1}^{(m+1) N}\left[u_{1}(n) \cdot S_{I}(n-i)+u_{2}(n) \cdot S_{Q}(n-i)\right] \\
& \cdot C_{P, 1}(n-i) \\
w_{i, 2}(m)= & \sum_{n=m N+1}^{(m+1) N}\left[u_{2}(n) \cdot S_{I}(n-i)-u_{1}(n) \cdot S_{Q}(n-i)\right] \\
& \cdot C_{D, 1}(n-i) \\
w_{i, 3}(m)= & \sum_{n=m N+1}^{(m+1) N}\left[u_{1}(n) \cdot S_{I}(n-i)+u_{2}(n) \cdot S_{Q}(n-i)\right] \\
& \cdot C_{D, 1}(n-i) \\
w_{i, 4}(m)= & \sum_{n=m N+1}^{(m+1) N}\left[u_{2}(n) \cdot S_{I}(n-i)-u_{1}(n) \cdot S_{Q}(n-i)\right] \\
& \cdot\left[-C_{P, 1}(n-i)\right] .
\end{aligned}
$$

Assuming that the channel parameters of (9) and (10) remain constant over one symbol duration, i.e., $\beta_{k, l}^{(c)}(n)=\beta_{k, l}^{(c)}(m N)$ and $\beta_{k, l}^{(s)}(n)=\beta_{k, l}^{(s)}(m N)$ for $m N<n \leq(m+1) N, w_{i, 1}(m)$ can be rewritten as

$$
\begin{aligned}
& w_{i, 1}(m)=\sum_{k=1}^{K} \sum_{l=0}^{L-1} \beta_{k, l}^{(c)}(m N) \sum_{n=m N+1}^{(m+1) N} \\
& \cdot\left(\sqrt{g_{k}} \cdot S_{I}(n-l) C_{P, k}(n-l)-D_{k}(m)\right. \\
& \text { - } \left.S_{Q}(n-l) C_{D, k}(n-l)\right) \\
& \text { - } S_{I}(n-i) C_{P, 1}(n-i) \\
& -\sum_{k=1}^{K} \sum_{l=0}^{L-1} \beta_{k, l}^{(s)}(m N) \sum_{n=m N+1}^{(m+1) N} \\
& \cdot\left(\sqrt{g_{k}} \cdot S_{Q}(n-l) C_{P, k}(n-l)+D_{k}(m)\right. \\
& \text { - } \left.S_{I}(n-l) C_{D, k}(n-l)\right) \\
& \text { - } S_{I}(n-i) C_{P, 1}(n-i) \\
& +\sum_{k=1}^{K} \sum_{l=0}^{L-1} \beta_{k, l}^{(s)}(m N) \sum_{n=m N+1}^{(m+1) N} \\
& \cdot\left(\sqrt{g_{k}} \cdot S_{I}(n-l) C_{P, k}(n-l)-D_{k}(m)\right. \\
& \text { - } \left.S_{Q}(n-l) C_{D, k}(n-l)\right) \\
& \text { - } S_{Q}(n-i) C_{P, 1}(n-i) \\
& +\sum_{k=1}^{K} \sum_{l=0}^{L-1} \beta_{k, l}^{(c)}(m N) \sum_{n=m N+1}^{(m+1) N} \\
& \cdot\left(\sqrt{g_{k}} \cdot S_{Q}(n-l) C_{P, k}(n-l)+D_{k}(m)\right. \\
& \text { - } \left.S_{I}(n-l) C_{D, k}(n-l)\right) \\
& \text { - } S_{Q}(n-i) C_{P, 1}(n-i) \\
& (m+1) N \\
& +\sum_{n=m N+1} \eta_{1}(n) S_{I}(n-i) C_{P, 1}(n-i) \\
& (m+1) N \\
& +\sum_{n=m N+1}^{m+1) N} \eta_{2}(n) S_{Q}(n-i) C_{P, 1}(n-i) \\
& =E_{i, 1}(m)+I_{i, 1}(m)+N_{i, 1}(m)
\end{aligned}
$$


then $w_{i, 1}(m)$ consists of three components:

1) desired signal component $E_{i, 1}(m)$;

2) interference component $I_{i, 1}(m)$;

3) background AWGN component $N_{i, 1}(m)$.

The desired signal component $E_{i, 1}(m)$ is the pilot component of the reference user in the reference path (i.e., $k=1$ and $l=i$ ) and is given by

$$
\begin{aligned}
E_{i, 1}(m)= & 2 N \beta_{1, i}^{(c)}(m N) \sqrt{g_{1}} \\
= & N \alpha_{1, i}(m N) R\left[\bar{\tau}_{1, i}(m N)\right] \\
& \cdot \cos \left[\varphi_{1, i}(m N)\right] \sqrt{g_{1} E_{D, 1}} .
\end{aligned}
$$

For the reference path (i.e., $\left.\bar{\tau}_{1, i}(m N)=0\right), R\left[\bar{\tau}_{1, i}(m N)\right]=1$. Therefore, a good estimate of the channel parameter $\left\{\alpha_{1, i}(t) \cos \varphi_{1, i}(t)\right\}$ can be derived from $E_{i, 1}(m)$, which is approximated by

$$
E_{i, 1}(m)=N \alpha_{1, i}(m N) \cos \left[\varphi_{1, i}(m N)\right] \sqrt{g_{1} E_{D, 1}} .
$$

The interference component $I_{i, 1}(m)$ is due to the cross-correlation with other users' spreading sequences, the autocorrelation of self-signal in other paths and the cross talk with quadrature components. Accurate evaluation of this interference component should involve all correlations in the specific sequence set. For simple analysis, it is assumed that all sequences are purely random binary sequences, except the case that $C_{p, 1}(n-$ $i)$ and $C_{D, 1}(n-i)$ are orthogonal. Thus, $I_{i, 1}(m)$ can be written as

$$
\begin{aligned}
& I_{i, 1}(m)=\sum_{k=1}^{K} \sum_{\substack{l=0 \\
\text { if } k=1, l \neq i}}^{L-1} \beta_{k, l}^{(c)}(m N) \cdot \sqrt{g_{k}} \cdot c_{k, l}^{(1)}(N) \\
& -\sum_{k=1}^{K} \sum_{l=0}^{L-1} \beta_{k, l}^{(c)}(m N) \cdot D_{k}(m) \cdot c_{k, l}^{(2)}(N) \\
& -\sum_{k=1}^{K} \sum_{l=0}^{L-1} \beta_{k, l}^{(s)}(m N) \cdot \sqrt{g_{k}} \cdot c_{k, l}^{(3)}(N) \\
& -\sum_{k=1}^{K} \sum_{\substack{l=0 \\
\text { if } k=1, l \neq i}}^{L-1} \beta_{k, l}^{(s)}(m N) D_{k}(m) c_{k, l}^{(4)}(N) \\
& +\sum_{k=1}^{K} \sum_{l=0}^{L-1} \beta_{k, l}^{(s)}(m N) \cdot \sqrt{g_{k}} \cdot c_{k, l}^{(5)}(N) \\
& -\sum_{k=1}^{K} \sum_{\substack{l=0 \\
\text { if } k=1, l \neq i}}^{L-1} \beta_{k, l}^{(s)}(m N) D_{k}(m) c_{k, l}^{(6)}(N) \\
& +\sum_{k=1}^{K} \sum_{\substack{l=0 \\
\text { if } k=1, l \neq i}}^{L-1} \beta_{k, l}^{(c)}(m N) \cdot \sqrt{g_{k}} \cdot c_{k, l}^{(7)}(N) \\
& +\sum_{k=1}^{K} \sum_{l=0}^{L-1} \beta_{k, l}^{(c)}(m N) \cdot D_{k}(m) \cdot c_{k, l}^{(8)}(N)
\end{aligned}
$$

where $c_{k, l}^{(\cdot)}(N)$ is the discrete aperiodic correlation of different sequence combinations with length $N,\left\{c_{k, l}^{(\cdot)}(N)\right\}$ are independent identical distributed (i.i.d.) random variables with zeromean, and variance $\operatorname{var}\left\{c_{k, l}^{(\cdot)}(N)\right\}=E\left\{\left[c_{k, l}^{(\cdot)}(N)\right]^{2}\right\}=N$. For different user $k$ and different path $l$, all the fading amplitudes $\alpha_{k, l}(m N)$ are independent, chip-timing errors $\bar{\tau}_{k, l}(m N)$ are i.i.d. random variables uniformly distributed in $\left(0, T_{c}\right]$, carrier phase offsets $\varphi_{k, l}(m N)$ are i.i.d. random variables uniformly distributed in $(0,2 \pi]$, and user data $D_{k}(m)$ are i.i.d random binary variables with zero-mean and variance 1 . Thus, $I_{i, 1}(m)$ is a random variable conditioned on $\alpha_{1, i}(m N)$, with zero-mean and variance given by

$$
\begin{aligned}
& \operatorname{var}\left\{I_{i, 1}(m)\right\}=\frac{1}{2} \sum_{k=1}^{K} \sum_{\substack{l=0 \\
\text { if } k=1, l \neq i}}^{L-1} g_{k} E_{D, k} \cdot E\left\{\alpha_{k, l}^{2}(m N)\right\} \\
& \text { - } E\left\{\cos ^{2} \varphi_{k, l}(m N)\right\} \cdot E\left\{R^{2}\left[\bar{\tau}_{k, l}(m N)\right]\right\} \\
& \text { - } E\left\{\left[c_{k, l}^{(\cdot)}(N)\right]^{2}\right\}+\frac{1}{2} \sum_{k=1}^{K} \sum_{l=0}^{L-1} E_{D, k} \\
& \text { - } E\left\{\alpha_{k, l}^{2}(m N)\right\} \cdot E\left\{\cos ^{2} \varphi_{k, l}(m N)\right\} \\
& \cdot E\left\{R^{2}\left[\bar{\tau}_{k, l}(m N)\right]\right\} \cdot E\left\{\left[c_{k, l}^{(\cdot)}(N)\right]^{2}\right\} \\
& \text { - } E\left\{D_{k}^{2}(m)\right\}+\frac{1}{2} \sum_{k=1}^{K} \sum_{l=0}^{L-1} g_{k} E_{D, k} \\
& \text { - } E\left\{\alpha_{k, l}^{2}(m N)\right\} \cdot E\left\{\sin ^{2} \varphi_{k, l}(m N)\right\} \\
& \cdot E\left\{R^{2}\left[\bar{\tau}_{k, l}(m N)\right]\right\} \cdot E\left\{\left[c_{k, l}^{(\cdot)}(N)\right]^{2}\right\} \text {. } \\
& +\frac{1}{2} \sum_{k=1}^{K} \sum_{\substack{l=0 \\
\text { if } k=1, l \neq i}}^{L-1} E_{D, k} E\left\{\alpha_{k, l}^{2}(m N)\right\} \\
& \text { - } E\left\{\sin ^{2} \varphi_{k, l}(m N)\right\} \cdot E\left\{R^{2}\left[\bar{\tau}_{k, l}(m N)\right]\right\} \\
& \text { - } E\left\{\left[c_{k, l}^{(\cdot)}(N)\right]^{2}\right\} E\left\{D_{k}^{2}(m)\right\} \\
& =\Delta \frac{N}{2} \sum_{k=1}^{K}\left(1+g_{k}\right) E_{D, k} \\
& \text {. } \sum_{\substack{l=0 \\
\text { if } k=1, l \neq i}}^{L-1} E\left\{\alpha_{k, l}^{2}(m N)\right\} \\
& +\Delta \frac{N}{4} E_{D, 1}\left(1+g_{1}\right) \alpha_{1, i}^{2}(m N) \\
& =\Delta \frac{N}{2} \sum_{k=1}^{K} E_{k} \sum_{\substack{l=0 \\
\text { if } k=1, l \neq i}}^{L-1} E\left\{\alpha_{k, l}^{2}(m N)\right\} \\
& +\Delta \frac{N}{4} E_{1} \alpha_{1, i}^{2}(m N)
\end{aligned}
$$

where $\Delta=E\left\{R^{2}\left[\bar{\tau}_{k, l}(m N)\right]\right\}$ is related to the frequency transfer function of pulse-shaping filter, which is given by

$$
\begin{aligned}
\Delta=E\left\{R^{2}\left[\bar{\tau}_{k, l}(m N)\right]\right\}=\frac{2}{3} \\
\\
\quad \text { for rectangular pulse shaping filter }
\end{aligned}
$$

$\operatorname{var}\left\{I_{i, 1}(m)\right\}$ in (20) is related to the instantaneous channel-fading amplitude $\alpha_{1, i}(m N)$ of the $i$ th path of the reference user. However, the contribution of $\alpha_{1, i}(m N)$ to the variance is very small when $K L$ is large, even for small $K$. In the extreme case $(K=1)$, the power ratio of the $i$ th path to other paths is $(1 / 2) E\left\{\alpha_{1, i}^{2}(m N)\right\} / \sum_{l=0, l \neq i}^{L-1} E\left\{\alpha_{k, l}^{2}(m N)\right\}$, 
which is very small when $L \gg 1$. Since adding $\alpha_{1, i}(m N)$ to $\operatorname{var}\left\{I_{i, 1}(m)\right\}$ makes the analysis troublesome, for simple analysis, the effect of $\alpha_{1, i}(m N)$ is averaged in the variance. Therefore, $\operatorname{var}\left\{I_{i, 1}(m)\right\}$ is approximated by

$$
\begin{aligned}
\operatorname{var}\left\{I_{i, 1}(m)\right\}= & \Delta \frac{N}{2} \sum_{k=1}^{K} E_{k} \sum_{\substack{l=0 \\
\text { if } k=1, l \neq i}}^{L-1} E\left\{\alpha_{k, l}^{2}(m N)\right\} \\
& +\Delta \frac{N}{4} E_{1} E\left\{\alpha_{1, i}^{2}(m N)\right\} \\
\approx & \Delta \frac{N}{2} \sum_{k=1}^{K} E_{k} \sum_{l=0}^{L-1} E\left\{\alpha_{k, l}^{2}(m N)\right\} \\
= & \frac{\Delta}{2} N K E \Omega_{T}
\end{aligned}
$$

where for the sake of simple analysis, it is assumed that $g_{k}=$ $g_{1}=g, E_{k}=E_{1}=E . \Omega_{T}$ is the channel parameter given by

$\Omega_{T}=\Omega_{T, k}=\sum_{l=0}^{L-1} E\left\{\alpha_{k, l}^{2}(m N)\right\}, \quad k=1,2, \ldots K$.

The AWGN component $N_{i, 1}(m)$ is a zero-mean Gaussian variable with variance

$$
\operatorname{var}\left\{N_{i, 1}(m)\right\}=2 N \cdot \frac{\eta_{0}}{2}=N \eta_{0} .
$$

Therefore, conditioned on $\alpha_{1, i}(m N) \cos \left[\varphi_{1, i}(m N)\right]$, $w_{i, 1}(m)$ is a random variable with mean $E_{i, 1}(m)$, given by (18), and variance $\operatorname{var}\left\{I_{i, 1}(m)\right\}+\operatorname{var}\left\{N_{i, 1}(m)\right\}$, i.e.,

$$
\operatorname{var}\left\{w_{i, 1}(m)\right\}=N\left(\eta_{0}+\frac{\Delta}{2} K E \Omega_{T}\right) .
$$

Similarly, $w_{i, 2}(m), w_{i, 3}(m)$, and $w_{i, 4}(m)$ can be approximated as conditional random variables, with same variance as $w_{i, 1}(m)$ but different means

$$
\begin{aligned}
& E\left\{w_{i, 2}(m)\right\}=N D_{1}(m) \alpha_{1, i}(m N) \cos \left[\varphi_{1, i}(m N)\right] \sqrt{E_{D}} \\
& E\left\{w_{i, 3}(m)\right\}=N D_{1}(m) \alpha_{1, i}(m N) \sin \left[\varphi_{1, i}(m N)\right] \sqrt{E_{D}} \\
& E\left\{w_{i, 4}(m)\right\}=N \alpha_{1, i}(m N) \sin \left[\varphi_{1, i}(m N)\right] \sqrt{g E_{D}}
\end{aligned}
$$

where $E_{D}=E_{D, 1}=E_{1} /\left(1+g_{1}\right)=E /(1+g)$ is the chip energy for data channel of the reference user.

From analysis above, it has been seen that in such wideband CDMA systems, the channel estimation using pilot symbols suffers from multiaccess and multipath interference. In most publications on coherent demodulation, perfect channel estimation is assumed for the purpose of simplification. However, this assumption does not stand in our study, and such estimation error is considered in the following performance analysis.

Because the variance (error) of this channel estimation is nontrivial and has direct contribution to the decision noise (as it will be shown in the following analysis), it must be suppressed. A simple way to reduce the estimation error is to use a low pass filter (LPF), as shown in Fig. 3. The interference from multiaccess and multipath can be modeled as white noise with no correlation between successive symbols and with a wide spectrum allocation of the chip rate $R_{c}=1 / T_{c}$. Therefore, LPFs with a cut-off frequency much smaller than $R_{c}$ should be able to reduce much noise on $w_{i, 1}(m)$ and $w_{i, 4}(m)$. In our study, a $N_{P}$-tap ( $N_{P}$ can be either even or odd numbers) FIR filter is adopted to suppress the noise, as shown in Fig. 3. In order to assure that the channel parameters remain relatively constant over $N_{P}$ symbol duration, the cut-off frequency of the filter should be much larger than the maximum Doppler frequency $f_{d}$, i.e., $R_{b} / N_{p}=R_{c} /\left(N_{p} N\right) \gg f_{d}$, where $R_{b}$ is the channel data rate. Higuchi et al. [12] have shown by both simulations and experiments that system performance is very stable when the window size of the FIR filter is two time slots (around $1.3 \mathrm{~ms}$ ) for the Doppler frequency in the range of 0 to $200 \mathrm{~Hz}$. Note that the cut-off frequency of its FIR filter is $1 / 1.3 \mathrm{~ms} \approx 800 \mathrm{~Hz}$, which is much larger than the maximum Doppler frequency of $200 \mathrm{~Hz}$.

It is worth noting here that in the uplink of wideband CDMA standards in 3GPP [4], two different spreading factors (SFs) are used for pilot and data channels, respectively. The SF $N_{1}$ of the pilot channel is fixed to be 256 , while the SF $N_{2}$ of data channel is varied from 4 to 256 . The channel estimate that is derived from one long pilot symbol corresponds to $N_{1} / N_{2}$ data symbols, depending on data rate. In this case, it is equivalent to say that the length of the FIR filter for 3GPP is $N_{P} /\left(N_{1} / N_{2}\right)$.

The statistic of channel estimation after the averaging LPF can be written as follows:

$$
\hat{w}_{i, 1}(m)=\frac{1}{N_{P}} \sum_{j=-\left[\left(N_{p}-1\right) / 2\right]}^{\left[N_{p} / 2\right]} w_{i, 1}(m+j) .
$$

Assuming that the variances of successive $w_{i, 1}(m)$ for different $m$ are independent to each other, one obtains

$$
\begin{aligned}
E\left\{\hat{w}_{i, 1}(m)\right\} & =E\left\{w_{i, 1}(m)\right\} \\
\operatorname{var}\left\{\hat{w}_{i, 1}(m)\right\} & =\frac{1}{N_{P}} \operatorname{var}\left\{w_{i, 1}(m)\right\} .
\end{aligned}
$$

The estimation error is greatly reduced by the LPF when $N_{p} \gg$ 1 . Similarly, the estimation error of $w_{i, 4}(m)$ can be reduced significantly as well. On the other hand, because the variables $w_{i, 2}(m)$ and $w_{i, 3}(m)$ contain unknown data for successive $m$, LPF may not be used to reduce the variances of these two statistics.

The output of the $i$ th branch in RAKE receiver is given by

$$
z_{i}(m)=\hat{w}_{i, 1}(m) \cdot w_{i, 2}(m)+w_{i, 3}(m) \cdot \hat{w}_{i, 4}(m) .
$$

From (16), it is seen that the sub-branch outputs are actually the sums of a lot of independent random variables; thus, $z_{i}(m)$ is in the form of $\left(\sum_{i} x_{i}\right) \cdot\left(\sum_{j} y_{j}\right)=\sum_{i} \sum_{j} x_{i} y_{j}$. That is, $z_{i}(m)$ consists of the sum of many independent random variables. Therefore, $z_{i}(m)$ can be approximated as a conditional Gaussian variable. Since $\operatorname{Re}(\cdot)$ and $\operatorname{Im}(\cdot)$ in Fig. 3 are independent, and $C_{P, 1}$ and $C_{D, 1}$ are orthogonal, $\hat{w}_{i, 1}(m), w_{i, 2}(m)$, $w_{i, 3}(m)$ and $\hat{w}_{i, 4}(m)$ are mutually independent to each other; therefore, the mean of $z_{i}(m)$ is given by

$$
\begin{aligned}
E\left\{z_{i}(m)\right\}= & E\left\{\hat{w}_{i, 1}(m)\right\} \cdot E\left\{w_{i, 2}(m)\right\} \\
& +E\left\{w_{i, 3}(m)\right\} \cdot E\left\{\hat{w}_{i, 4}(m)\right\} \\
= & N^{2} D_{1}(m) \alpha_{1, i}^{2}(m N) E_{D} \sqrt{g} .
\end{aligned}
$$


The variance of $z_{i}(m)$ is given by

$$
\begin{aligned}
\operatorname{var}\left\{z_{i}(m)\right\} & \\
= & E\left\{\left[\hat{w}_{i, 1}(m) \cdot w_{i, 2}(m)+w_{i, 3}(m) \cdot \hat{w}_{i, 4}(m)\right]^{2}\right\} \\
& -E^{2}\left\{\hat{w}_{i, 1}(m) \cdot w_{i, 2}(m)+w_{i, 3}(m) \cdot \hat{w}_{i, 4}(m)\right\} \\
= & E^{2}\left\{\hat{w}_{i, 1}(m)\right\} \cdot \operatorname{var}\left\{w_{i, 2}(m)\right\} \\
& +E^{2}\left\{w_{i, 2}(m)\right\} \cdot \operatorname{var}\left\{\hat{w}_{i, 1}(m)\right\} \\
& +E^{2}\left\{w_{i, 3}(m)\right\} \cdot \operatorname{var}\left\{\hat{w}_{i, 4}(m)\right\} \\
& +E^{2}\left\{\hat{w}_{i, 4}(m)\right\} \cdot \operatorname{var}\left\{w_{i, 3}(m)\right\} \\
& +\operatorname{var}\left\{\hat{w}_{i, 1}(m)\right\} \cdot \operatorname{var}\left\{w_{i, 2}(m)\right\} \\
& +\operatorname{var}\left\{w_{i, 3}(m)\right\} \cdot \operatorname{var}\left\{\hat{w}_{i, 4}(m)\right\} \\
= & \left(g+\frac{1}{N_{P}}\right) \alpha_{1, i}^{2}(m N) N^{3} E_{D} \cdot\left(\eta_{0}+\frac{\Delta}{2} K E \Omega_{T}\right) \\
& +\frac{2 N^{2}}{N_{P}}\left(\eta_{0}+\frac{\Delta}{2} K E \Omega_{T}\right)^{2} .
\end{aligned}
$$

In the case of perfect channel estimation, i.e., $\operatorname{var}\left\{\hat{w}_{i, 1}(m)\right\}=\operatorname{var}\left\{\hat{w}_{i, 4}(m)\right\}=0$ or $N_{P} \rightarrow \infty$ in (34), the mean of $z_{i}(m)$ remains unchanged, but the variance reduces to $\left.\operatorname{var}\left\{z_{i}(m)\right\}\right|_{N_{p} \rightarrow \infty}=g \alpha_{1, i}^{2}(m N) N^{3} E \cdot\left(\eta_{0}+\frac{\Delta}{2} K E \Omega_{T}\right)$.

Assuming that the fading of each path is independent of each other and the output in each branch of the RAKE receiver is independent of each other, the summed output $Z(m)=\sum_{i=0}^{M-1} z_{i}(m)$, where $M$ is the number of branches, is a Gaussian variable with mean and variance given by

$$
\begin{aligned}
E & \left\{\left.Z(m)\right|_{\alpha_{1, i}(m N), i=0,1, \ldots, M-1}\right\} \\
& =E\left\{\left.Z(m)\right|_{\zeta}\right\} \\
& =\sum_{i=0}^{M-1} E\left\{z_{i}(m)\right\} \\
& =E_{D} N^{2} \sqrt{g} \cdot D_{1}(m) \cdot \sum_{i=0}^{M-1} \alpha_{1, i}^{2}(m N) \\
& =E_{D} N^{2} \sqrt{g} \cdot D_{1}(m) \cdot \zeta
\end{aligned}
$$

where

$$
\zeta=\sum_{i=0}^{M-1} \alpha_{1, i}^{2}(m N)
$$

and

$$
\begin{aligned}
\operatorname{var} & \left\{\left.Z(m)\right|_{\alpha_{1, i}(m N), i=0,1, \ldots, M-1}\right\} \\
= & \operatorname{var}\left\{\left.Z(m)\right|_{\zeta}\right\} \\
= & \sum_{i=0}^{M-1} \operatorname{var}\left\{z_{i}(m)\right\} \\
= & \left(g+\frac{1}{N_{P}}\right) N^{3} E_{D} \cdot \sum_{i=0}^{M-1} \alpha_{1, i}^{2}(m N)\left(\eta_{0}+\frac{\Delta}{2} K E \Omega_{T}\right) \\
& +\frac{2 N^{2}}{N_{P}} \sum_{i=0}^{M-1}\left(\eta_{0}+\frac{\Delta}{2} K E \Omega_{T}\right)^{2} \\
= & \left(g+\frac{1}{N_{P}}\right) N^{3} E_{D} \zeta\left(\eta_{0}+\frac{\Delta}{2} K E \Omega_{T}\right) \\
& +\frac{2 M N^{2}}{N_{P}}\left(\eta_{0}+\frac{\Delta}{2} K E \Omega_{T}\right)^{2} .
\end{aligned}
$$

Therefore, conditioned on the instantaneous multipath fading amplitude $\zeta$ of the reference user, the bit error probability is given by (39), shown at the bottom of the page, where $N E / \eta_{0}$ is the signal-to-noise ratio (SNR), $Q(x)=\int_{x}^{\infty}(1 / \sqrt{2 \pi}) e^{-y^{2} / 2} d y$, and

$$
\begin{aligned}
& a=(1+g)\left(1+\frac{1}{g N_{P}}\right)\left(\frac{1}{\mathrm{SNR}}+\frac{\Delta}{2} \Omega_{T} \frac{K}{N}\right) \\
& b=\frac{2 M(1+g)^{2}}{g N_{P}}\left(\frac{1}{\mathrm{SNR}}+\frac{\Delta}{2} \Omega_{T} \frac{K}{N}\right)^{2} .
\end{aligned}
$$

For a specific channel model, $\zeta$ is a random variable with probability density function $p(\zeta)$. The average bit error probability $P_{e}$ can be obtained by averaging $P_{e}(\zeta)$ over $\zeta$, i.e.,

$$
\begin{aligned}
P_{e}= & \int_{0}^{\infty} P_{e}(\zeta) p(\zeta) d \zeta \\
= & \underbrace{\int_{0}^{\infty} \int_{0}^{\infty} \cdots \int_{0}^{\infty}}_{M} P_{e}\left(\sum_{i=0}^{M-1} \alpha_{1, i}^{2}\right) \\
& \cdot p\left(\alpha_{1,0}, \alpha_{1,1} \cdots \alpha_{1, M-1}\right) d \alpha_{1,0} d \alpha_{1,1} \cdots d \alpha_{1, M-1}
\end{aligned}
$$

where $p\left(\alpha_{1,0}, \alpha_{1,1} \ldots \alpha_{1, M-1}\right)$ is the joint probability density function of multipath fading amplitudes.

Equations (39) and (42) give a complete bit error rate (BER) performance evaluation of the wideband CDMA system with

$$
\begin{aligned}
P_{e}(\zeta) & =Q\left(\frac{E\left\{\left.Z(m)\right|_{\zeta}\right\}}{\sqrt{\operatorname{var}\left\{\left.Z(m)\right|_{\zeta}\right\}}}\right) \\
& =Q\left(\left\{\begin{array}{l}
\left.\left.\frac{(1+g)}{\zeta}\left(1+\frac{1}{g N_{P}}\right)\left(\frac{1}{N E / \eta_{0}}+\frac{\Delta}{2} \Omega_{T} \frac{K}{N}\right)\right\}^{-1 / 2}\right) \\
+\frac{1}{\zeta^{2}} \frac{2 M(1+g)^{2}}{g N_{P}}\left(\frac{1}{N E / \eta_{0}}+\frac{\Delta}{2} \Omega_{T} \frac{K}{N}\right)^{2}
\end{array}\right\}\right) \\
& =Q\left(\frac{\zeta}{\sqrt{a \zeta+b}}\right)
\end{aligned}
$$


user dedicated pilot symbols. However, (42) is generally too complicated to be computed, and the probability density function (pdf) $p(\zeta)$ is unobtainable if the fading in each path is to be chosen arbitrarily. In order to simplify the numerical evaluation, it is assumed that each user has independent but the same fading characteristics. The amplitude fading in each path is assumed to be Nakagami-distributed since the Nakagami distribution is more versatile and more adequate to describe different fading situations. For example, Nakagami fading can closely represent Rayleigh and Rician fading under appropriate parameters [8], [10]. The Nakagami pdf of $\alpha_{1, i}=\alpha_{i}$ is given by

$$
p\left(\alpha_{i}\right)=2\left(\frac{m_{i}}{\Omega_{i}}\right)^{m_{i}} \frac{\alpha_{i}^{2 m_{i}-1}}{\Gamma\left(m_{i}\right)} \exp \left(-\frac{m_{i}}{\Omega_{i}} \alpha_{i}^{2}\right), \quad \alpha_{i} \geq 0
$$

where $m_{i}=\left(\Omega_{i}^{2} /\left(E\left\{\left(\alpha_{i}^{2}-\Omega_{i}\right)^{2}\right\}\right)\right), \Omega_{i}=E\left\{\alpha_{i}^{2}\right\}=\Omega_{0} e^{-i \delta}$, and $\delta$ is the rate of exponential decay of the multipath intensity profile (MIP). $\Gamma(x)=\int_{0}^{\infty} t^{x-1} e^{-t} d t$ is the Gamma function. If the Nakagami distribution in each path is such that the ratio $\left(m_{i} / \Omega_{i}\right)$ is the same for all paths, it can be shown that $\zeta$ has the gamma pdf [15]

$$
p(\zeta)=\left(\frac{m_{T}}{\Omega_{T}}\right)^{m_{T}} \frac{\zeta^{m_{T}-1}}{\Gamma\left(m_{T}\right)} \exp \left(-\frac{m_{T}}{\Omega_{T}} \zeta\right), \quad \zeta \geq 0
$$

where $\Omega_{T}=\sum_{i=0}^{M-1} \Omega_{i}=\sum_{i=0}^{M-1} E\left\{\alpha_{i}^{2}\right\}$, and $m_{T}=\sum_{i=0}^{M-1} m_{i}$. Submitting (44) into (42), the average bit error probability $P_{e}$ is given by

$$
\begin{aligned}
P_{e}= & \int_{0}^{\infty} P_{e}(\zeta) p(\zeta) d \zeta \\
= & \int_{0}^{\infty} Q\left(\frac{\zeta}{\sqrt{a \zeta+b}}\right) \cdot\left(\frac{m_{T}}{\Omega_{T}}\right)^{m_{T}} \\
& \cdot \frac{\zeta^{m_{T}-1}}{\Gamma\left(m_{T}\right)} \exp \left(-\frac{m_{T}}{\Omega_{T}} \zeta\right) d \zeta .
\end{aligned}
$$

In the case of perfect channel estimation, (45) has a known solution [8], [10]. In general, the integral of (45) can be computed numerically using computer software like MATLAB.

It is seen from (39)-(41) that for a given SNR and number of active users, the change of the power ratio $g$ has certain influence on the system performance. When $g$ is very large or very small, at least one of $a$ and $b$ will be large so that large error probability is caused. In other words, when $g$ is very large, the desired component [see (18) or (28)] in the pilot channel is strong (i.e., channel estimation quality is good), whereas the desired component [see (26) or (27)] in the data channel is weak. This will cause large error probability. On the other hand, when $g$ is very small, channel estimation quality is poor so that large error probability is caused as well. Therefore, there exists an optimal value of $g$, which minimizes the error probability or maximizes the signal-to-interference-plus-noise ratio. The op-

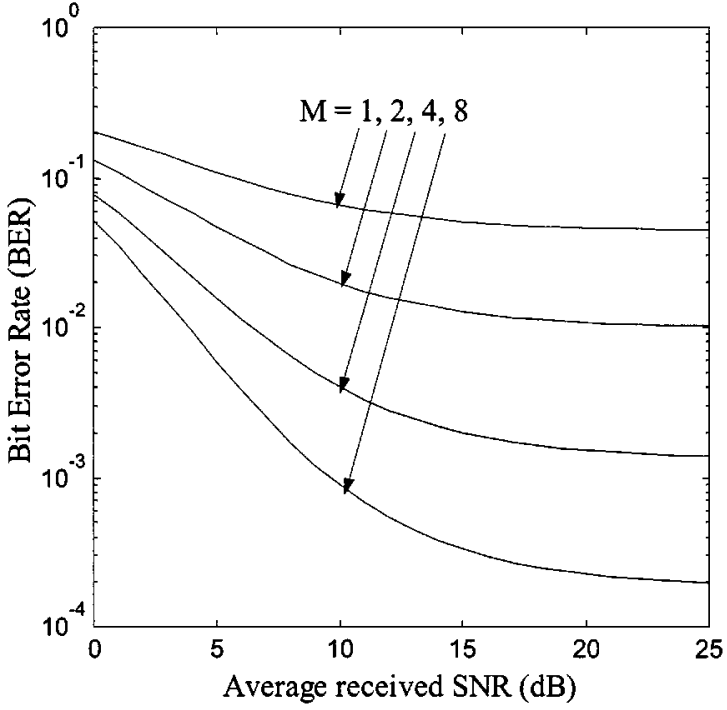

Fig. 4. BER versus average received SNR with different number of RAKE fingers.

timal $g$ is obtainable by minimizing (39). That is, by letting $d(a \zeta+b) / d g=0$, it is readily to have

$$
g_{\text {opt }}(\zeta)=\sqrt{\frac{2 M\left(\frac{1}{\mathrm{SNR}}+\frac{\Delta}{2} \Omega_{T} \frac{K}{N}\right)+\zeta}{2 M\left(\frac{1}{\mathrm{SNR}}+\frac{\Delta}{2} \Omega_{T} \frac{K}{N}\right)+\zeta \cdot N_{p}}} .
$$

Similar to the discussion of the average bit error probability, an average $g_{o p t}$ can be obtained by averaging $g_{o p t}(\zeta)$ over $\zeta$, i.e.,

$$
\begin{aligned}
g_{\text {opt }} & =\int_{0}^{\infty} g_{\text {opt }}(\zeta) p(\zeta) d \zeta \\
& =\int_{0}^{\infty} g_{\text {opt }}(\zeta) \cdot\left(\frac{m_{T}}{\Omega_{T}}\right)^{m_{T}} \frac{\zeta^{m_{T}-1}}{\Gamma\left(m_{T}\right)} \exp \left(-\frac{m_{T}}{\Omega_{T}} \zeta\right) d \zeta .
\end{aligned}
$$

\section{NUMERICAL RESUlTS}

In this section, the effects of different system parameters on the BER performance of the wideband CDMA system with complex spreading and imperfect channel estimation are investigated by numerical calculations. The fading is assumed to be Nakagami distributed, as noted in Section III. The exponential decay rate of the MIP $\delta$ is 0.2 . The ratio $m_{l} / \Omega_{l}$ is 4 , and $\Omega_{0}$ is set as 1 for normalization. The number of multipaths $L$ is 8 , and the spreading factor $N$, i.e., number of chips per data symbol, is fixed to be 64. Unless noted otherwise, the number of RAKE fingers $M$ is 4 . The LPF length is $N_{P}=32$, the number of active users in the system $K$ is 10 , the power ratio of in-phase and quadrature branches $g$ is 0.3 , and the SNR is $15 \mathrm{~dB}$.

Fig. 4 illustrates the BER as a function of average received SNR for different number of RAKE fingers, i.e., $M=1,2,4,8$. It can be seen that the RAKE receiver takes advantage of the multipath and gives better performance when the number of fingers increases. For the case of $M=4$, 


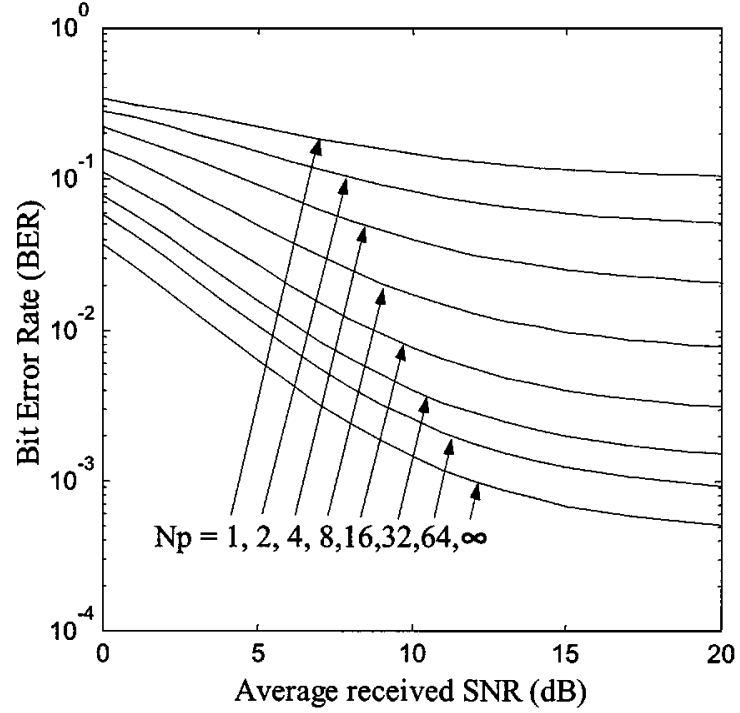

Fig. 5. BER versus average received SNR with different number of taps of LPFs.

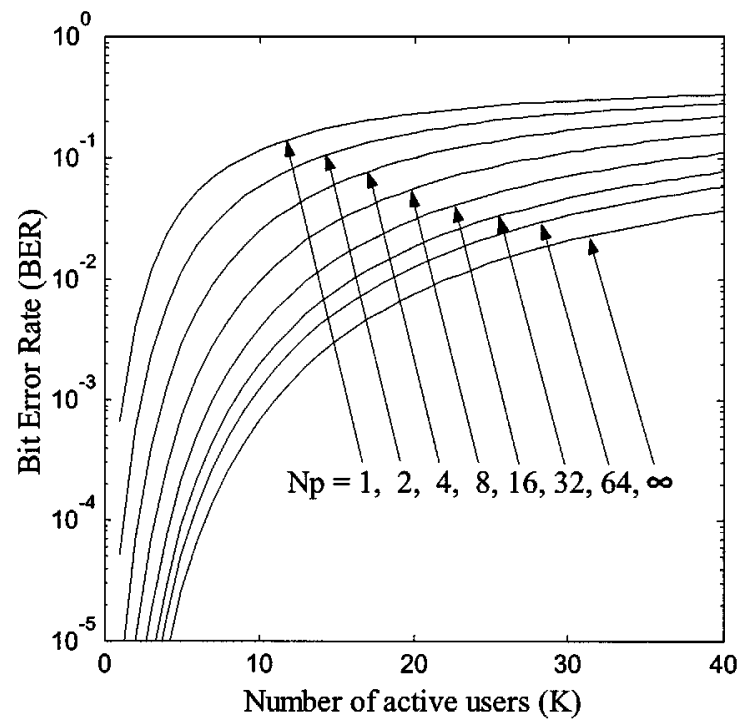

Fig. 6. BER versus number of users.

$\mathrm{BER} \approx 10^{-2}$ can be obtained when $\mathrm{SNR}=8 \mathrm{~dB}$. If channel coding is used, acceptable BER performance can be achieved.

Fig. 5 shows the BER versus average received SNR for different number of taps $N_{P}$ of LPF. The last curve, where $N_{P}=$ $\infty$, corresponds to the case of perfect channel estimation. From the analysis (39)-(41), it is seen that the increase of $N_{P}$ will increase the system performance. This is because the increase of the number of taps of LPFs decreases the estimation variance. It can be seen from the figure that using larger number of taps can improve performance significantly. A similar property can be found in Fig. 6, which shows the BER as a function of the number of active users $K$. It is clearly shown that the increment of $N_{P}$ always has positive effect to the system performance. For a given BER of $10^{-2}$, doubling the number of taps means the increase of capacity by three or four users. However, the value of $N_{P}$ is subject to the constraint that the channel parameters

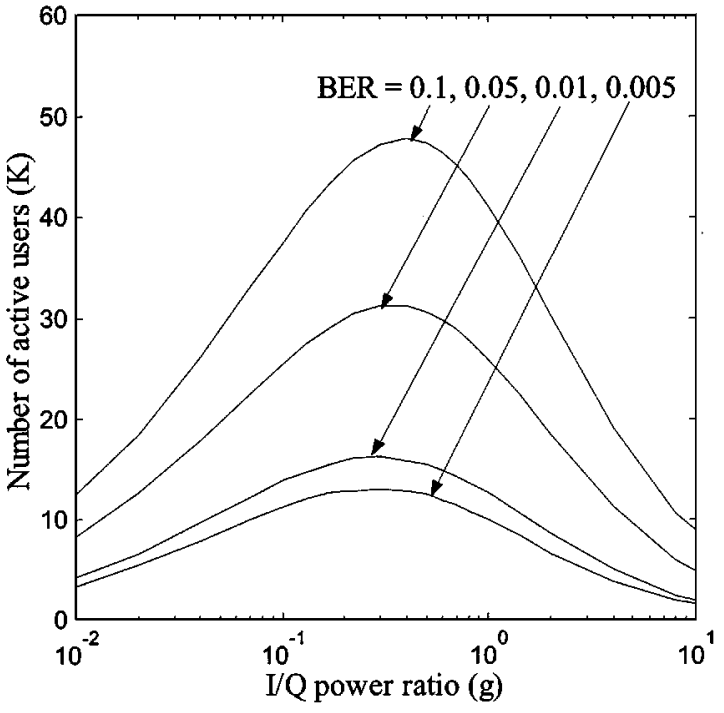

Fig. 7. Number of users versus I/Q power ratio with BER.

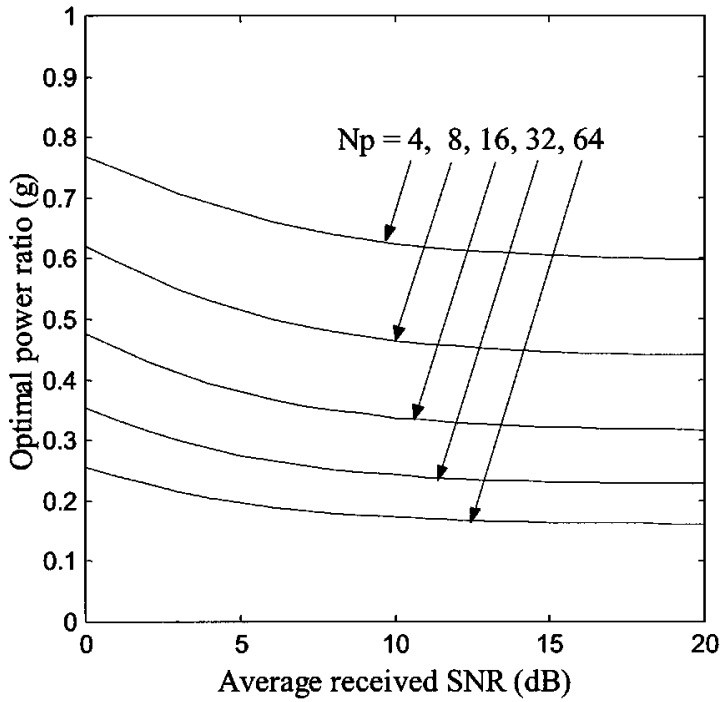

Fig. 8. Optimal power ratio versus average received SNR with different number of taps of LPFs.

should be kept constant over $N_{P}$ symbols time period. That is, $N_{P}$ cannot be chosen too large.

Fig. 7 illustrates the effects of I/Q power ratio $g$ to the system capacity (number of active users, $K$ ). It is seen from the figure that for a given BER, when $g$ is small, the increment of $g$ increases the system capacity significantly, whereas the capacity decreases when $g$ increases beyond its optimal value. One can find that the optimal values are slightly different for different BER level. Under the specified system parameters (i.e., SNR = $15 \mathrm{~dB}, N_{P}=32$, etc.), the optimal value for $\mathrm{BER}=0.1$ is about 0.4 , while it is about 0.3 for BER $=0.005$. The optimal power ratio $g_{\mathrm{opt}}$, given by (47), is plotted in Fig. 8 as a function of average SNR with different number of taps of LPF. As shown in the figure, the increase of SNR monotonously decreases the value of $g_{\mathrm{opt}}$. However, the change is very slight when SNR $\gg 0 \mathrm{~dB}$, which implies that the SNR has little influence on $g_{\mathrm{opt}}$. It can be also seen from the figure that in- 


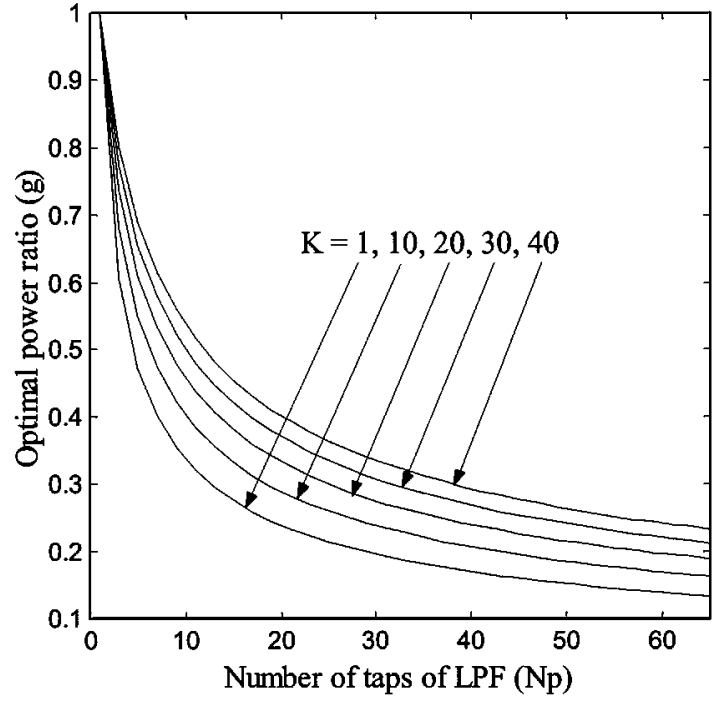

Fig. 9. Optimal power ratio versus number of taps of LPFs with different number of active user.

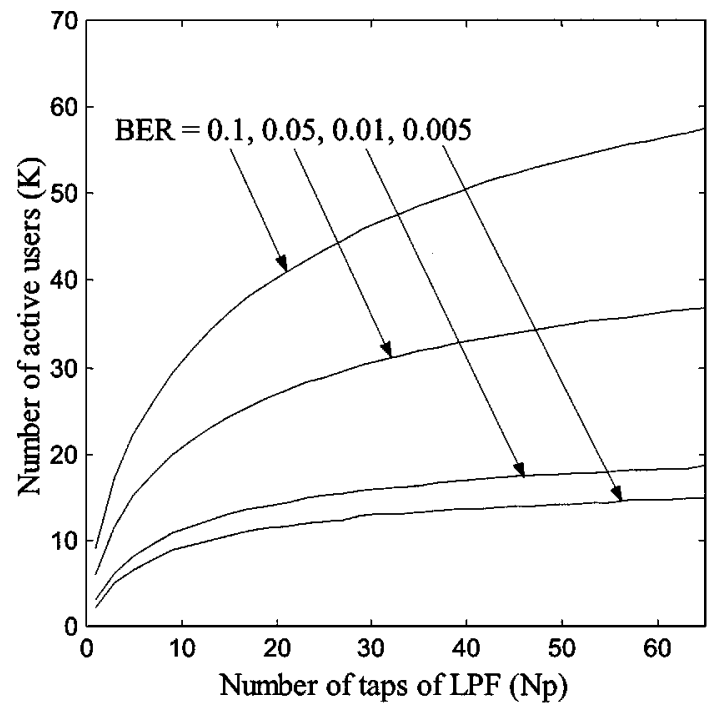

Fig. 10. Number of active users versus number of taps of LPFs.

creasing $N_{P}$ means decreasing the optimal value. This is because increasing $N_{P}$ improves the channel estimation quality so that the transmission power of the pilot can be reduced in order to maintain a given channel estimation error. This conclusion can also be obtained from Fig. 9, where $g_{\text {opt }}$ is shown as a function of number of taps of LPF $N_{P}$ for different number of active users. Similarly, the increase of $N_{P}$ monotonously decreases $g_{\text {opt }}$. The optimal value increases as the number of users increases. That is, when there are more users in the system, a larger portion of pilot power is preferred. It is worth noting that since SNR $=N E / \eta_{0}=15 \mathrm{~dB}$ is fixed, a larger $g$ means the increase of pilot power but the reduce of data channel power; thus, the total power of the system remains unchanged, i.e., the total interference of this cell to other cells is not raised.

Finally, the system capacity $K$ is shown in Fig. 10 as a function of the number of taps of the LPF for different values of BER. It can be clearly seen that the capacity can be significantly increased by the increase of $N_{P}$, especially when BER is small.
Actually, the relative large BERs can be pulled down to acceptable levels using channel coding-decoding (e.g., convoluational or Turbo coding). That is, system performance can be significantly improved by use of LPFs with a large value of $N_{P}$ in conjunction with channel coding.

\section{ENVELOPE OF COMPLEX AND DUAL-CHANNEL SPREAD SIGNAL}

One of the reasons to use complex spreading instead of dualchannel spreading is that the complex spreading scheme can reduce the peak-to-average ratio (PAR) of the modulated signal, thus improving the efficiency of RF power amplifier [3]. However, so far, there are no explicit explanations for this assertion. The PAR is a measurement of the signal envelope variation, which is defined as

$$
\operatorname{PAR}=\frac{\max \{|s(t)|\}}{\sqrt{E\left\{|s(t)|^{2}\right\}}}
$$

where $|s(t)|$ is the envelope of $s(t)$. For a QPSK signal $s(t)=$ $I(t) \cos \omega t-Q(t) \sin \omega t$, the envelope is effectively represented by $|s(t)|=\sqrt{I^{2}(t)+Q^{2}(t)}$. When PAR is evaluated, the max and mean operation in (48) should involve all kinds of symbol (chip) waveforms. The denominator of (48) is denoted as the root mean square (RMS) value of the signal, which corresponds to the average power transmitted. In practical measurements, the numerator of (48), i.e., the peak value of the envelope, is replaced by the 99.9 percentile of the amplitude distribution for reliable evaluation. Another convenient and useful parameter of the signal envelope variation is the normalized deviation (ND), which is defined as

$$
\mathrm{ND}=\frac{\sqrt{\operatorname{var}\{|s(t)|\}}}{\sqrt{E\left\{|s(t)|^{2}\right\}}}=\sqrt{1-\frac{E^{2}\{|s(t)|\}}{E\left\{|s(t)|^{2}\right\}}}
$$

This parameter effectively characterizes the deviation of signal envelope from its average value. The knowledge of the signal envelope variation helps to set the operating point as well as the back off of a RF power amplifier. Generally speaking, if the PAR and ND of a signal are small, the back off of the amplifier can be set smaller, which results in a higher power efficiency of the amplifier.

In (1), $S_{k}(t)$ can be rewritten as

$$
S_{k}(t)=I(t) \cos \left(\omega_{0} t+\phi_{k}\right)-Q(t) \sin \left(\omega_{0} t+\phi_{k}\right)
$$

where $I(t)$ and $Q(t)$ represent the baseband in-phase and quadrature components, and are given by

$$
\begin{aligned}
I(t)=\sqrt{E_{k}} \sum_{n=-\infty}^{\infty}\left[\sqrt{g_{k}} C_{P, k}(n) S_{I}(n)\right. \\
\left.\quad-D_{k}(m) C_{D, k}(n) S_{Q}(n)\right] h\left(t-n T_{c}\right) \\
=\sqrt{E_{k}} \sum_{n=-\infty}^{\infty} I_{n} h\left(t-n T_{c}\right)
\end{aligned}
$$


TABLE I

AlPhabet of Complex SPREADING AND DuAL-ChanNEL SPREADING

\begin{tabular}{|c|c|c|c|c|c|c|c|}
\hline$S_{i}(n)$ & $S_{Q}(n)$ & $\overline{C_{P, k}(n)}$ & $C_{D, k}(n)$ & $I_{n}$ & $Q_{n}$ & $I_{n}^{\prime}$ & $Q_{n}^{\prime}$ \\
\hline 1 & 1 & 1 & 1 & \multirow{2}{*}{$\sqrt{g_{k}}-1$} & \multirow{2}{*}{$\sqrt{g_{k}}+1$} & \multirow{2}{*}{$\sqrt{g_{k}}$} & \multirow[t]{2}{*}{1} \\
\hline-1 & -1 & -1 & -1 & & & & \\
\hline 1 & 1 & 1 & -1 & \multirow{2}{*}{$\sqrt{g_{k}}+1$} & \multirow{2}{*}{$\sqrt{g_{k}}-1$} & \multirow{2}{*}{$\sqrt{g_{k}}$} & \multirow{2}{*}{-1} \\
\hline-1 & -1 & -1 & 1 & & & & \\
\hline 1 & 1 & -1 & 1 & \multirow{2}{*}{$-\sqrt{g_{k}}-1$} & \multirow{2}{*}{$-\sqrt{g_{k}}+1$} & \multirow{2}{*}{$-\sqrt{g_{k}}$} & \multirow{2}{*}{1} \\
\hline-1 & -1 & 1 & -1 & & & & \\
\hline 1 & 1 & -1 & -1 & \multirow{2}{*}{$-\sqrt{g_{k}}+1$} & \multirow{2}{*}{$-\sqrt{g_{k}}-1$} & \multirow{2}{*}{$-\sqrt{g_{k}}$} & \multirow{2}{*}{-1} \\
\hline-1 & -1 & 1 & 1 & & & & \\
\hline 1 & -1 & 1 & 1 & \multirow{2}{*}{$\sqrt{g_{k}}+1$} & \multirow{2}{*}{$-\sqrt{g_{k}}+1$} & \multirow{2}{*}{$\sqrt{g_{k}}$} & \multirow{2}{*}{-1} \\
\hline-1 & 1 & -1 & -1 & & & & \\
\hline 1 & -1 & 1 & -1 & \multirow{2}{*}{$\sqrt{g_{k}}-1$} & \multirow{2}{*}{$-\sqrt{g_{k}}-1$} & \multirow{2}{*}{$\sqrt{g_{k}}$} & \multirow{2}{*}{1} \\
\hline-1 & 1 & -1 & 1 & & & & \\
\hline 1 & -1 & -1 & 1 & \multirow{2}{*}{$-\sqrt{g_{k}}+1$} & \multirow{2}{*}{$\sqrt{g_{k}}+1$} & \multirow{2}{*}{$-\sqrt{g_{k}}$} & \multirow{2}{*}{-1} \\
\hline-1 & 1 & 1 & -1 & & & & \\
\hline$\frac{1}{-1}$ & -1 & -1 & -1 & \multirow{2}{*}{$-\sqrt{g_{k}}-1$} & \multirow[t]{2}{*}{$\sqrt{g_{k}}-1$} & \multirow[t]{2}{*}{$-\sqrt{g_{k}}$} & \multirow[t]{2}{*}{1} \\
\hline & & 1 & 1 & & & & \\
\hline
\end{tabular}

$$
\begin{aligned}
Q(t)= & \sqrt{E_{k}} \sum_{n=-\infty}^{\infty}\left[\sqrt{g_{k}} C_{P, k}(n) S_{Q}(n)\right. \\
& \left.+D_{k}(m) C_{D, k}(n) S_{I}(n)\right] h\left(t-n T_{c}\right) \\
= & \sqrt{E_{k}} \sum_{n=-\infty}^{\infty} Q_{n} h\left(t-n T_{c}\right)
\end{aligned}
$$

where for simple notation, the definition of $h(t)$ is slightly different from (1). Here, $h(t)$ is defined to be symmetric, i.e., $\int_{-A T c / 2}^{A T c / 2} h^{2}(t) d t=1$, where $A \geq 1$. Note that the values of $I_{n}$ and $Q_{n}$ are correlated to each other. For dual-channel spreading, the transmitted signal is also given by (50) but with the baseband in-phase and quadrature components given by

$$
\begin{aligned}
I^{\prime}(t) & =\sqrt{2 E_{k}} \sum_{n=-\infty}^{\infty} \sqrt{g_{k}} C_{P, k}(n) S_{I}(n) h\left(t-n T_{c}\right) \\
& =\sqrt{2 E_{k}} \sum_{n=-\infty}^{\infty} I_{n}^{\prime} h\left(t-n T_{c}\right) \\
Q^{\prime}(t) & =\sqrt{2 E_{k}} \sum_{n=-\infty}^{\infty} D_{k}(m) C_{D, k}(n) S_{Q}(n) h\left(t-n T_{c}\right) \\
& =\sqrt{2 E_{k}} \sum_{n=-\infty}^{\infty} Q_{n}^{\prime} h\left(t-n T_{c}\right) .
\end{aligned}
$$

It can be seen that $I_{n}^{\prime}$ and $Q_{n}^{\prime}$ are independent from each other. Notice that both complex and dual-channel spread signals have the same average power. Given $D(m)=1$, all values of $\left(I_{n}\right.$, $\left.Q_{n}\right)$ and $\left(I_{n}^{\prime}, Q_{n}^{\prime}\right)$ are listed in Table I for all combinations of $C_{P, k}(n), C_{D, k}(n), S_{I}(n)$ and $S_{Q}(n)$.

As noted, the modulated signal envelope is effectively represented by $|s(t)|=\sqrt{I^{2}(t)+Q^{2}(t)}$. The relationships of the signal envelope with the modulating symbols $\left(I_{n}, Q_{n}\right)$ or $\left(I_{n}^{\prime}, Q_{n}^{\prime}\right)$ are very complicated if the baseband signals are pulse shaped. This is because pulse shaping not only limits the bandwidth of the transmitted signal, but also extends the waveform in time domain. Therefore, the waveform is no longer determined by current chip or symbol but subject to the effect of neighboring chips or symbols. In order to simplify the performance analysis, we have assumed that the chip waveform is limited to one chip

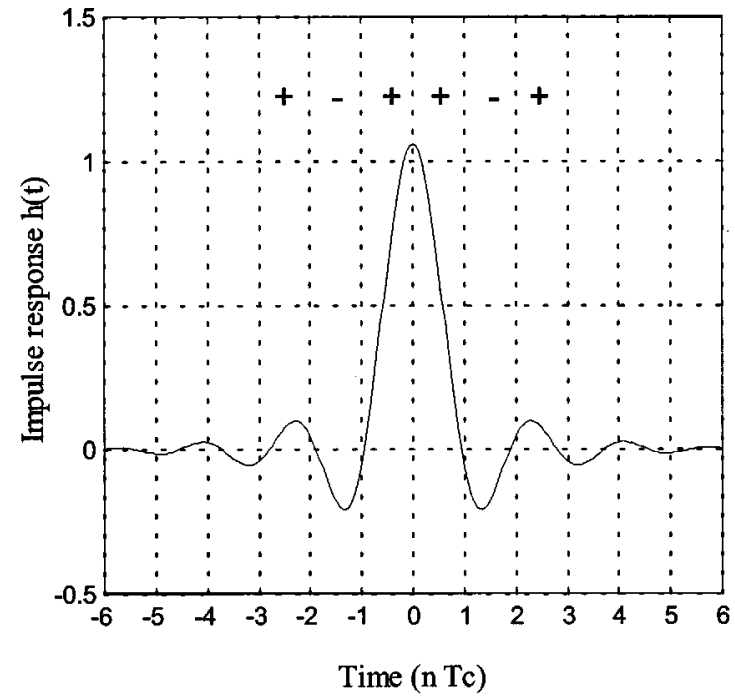

Fig. 11. Impulse response of square RRC filter with rolloff factor 0.22 .

TABLE II

SignAl ENVElope of Two SpREADING Schemes $(A=7)$

\begin{tabular}{c|c|c|c|c|c|c|c|c}
\hline \multirow{2}{*}{$g_{k}$} & \multicolumn{3}{|c|}{ dual-channel spreading } & \multicolumn{4}{c}{ complex spreading } \\
\cline { 2 - 9 } & RMS & MAX & VAR & ND & RMS & MAX & VAR & ND \\
\hline 1 & 1.4134 & 2.5358 & 0.1563 & 0.2797 & 1.4134 & 2.5358 & 0.1563 & 0.2797 \\
\hline 0.9 & 1.3776 & 2.4716 & 0.1488 & 0.2800 & 1.3776 & 2.4716 & 0.1484 & 0.2796 \\
\hline 0.8 & 1.3409 & 2.4057 & 0.1418 & 0.2809 & 1.3409 & 2.4057 & 0.1403 & 0.2794 \\
\hline 0.7 & 1.3031 & 2.3379 & 0.1357 & 0.2826 & 1.3031 & 2.3379 & 0.1321 & 0.2789 \\
\hline 0.6 & 1.2642 & 2.2681 & 0.1304 & 0.2856 & 1.2642 & 2.2681 & 0.1237 & 0.2782 \\
\hline 0.5 & 1.2240 & 2.1961 & 0.1262 & 0.2902 & 1.2240 & 2.1961 & 0.1151 & 0.2771 \\
\hline 0.4 & 1.1825 & 2.1216 & 0.1235 & 0.2971 & 1.1825 & 2.1216 & 0.1064 & 0.2759 \\
\hline 0.3 & 1.1395 & 2.0425 & 0.1227 & 0.3074 & 1.1395 & $\mathbf{2 . 0 4 2 5}$ & $\mathbf{0 . 0 9 7 8}$ & $\mathbf{0 . 2 7 4 4 5}$ \\
\hline 0.2 & 1.0948 & 1.9642 & 0.1248 & 0.3226 & 1.0948 & 1.9642 & 0.0897 & 0.2735 \\
\hline 0.1 & 1.0482 & 1.8806 & 0.1318 & 0.3463 & 1.0482 & 1.8806 & 0.0826 & 0.2741 \\
\hline 0 & 0.9994 & 1.7931 & 0.1544 & 0.3932 & 0.9994 & 1.7931 & 0.0781 & 0.2797 \\
\hline
\end{tabular}

interval, i.e., $A=1$. However, to evaluate the signal envelope under difference spreading schemes, it is necessary to extend the chip waveform to multiple chips, which is the situation in any practical systems.

As depicted in Fig. 11, the impulse reponse of the square root raised cosine filter with rolloff factor of 0.22 is truncated by a length of $A T_{c}$ for practical implementation. Actually, the chip waveform corresponding to each $\left(I_{n}, Q_{n}\right)$ pair for different $n$ is overlapped with each other. If $h(t)$ is truncated to the length $A T_{c}$, it can be observed that the current chip waveform is overlapped by its preceding $[A / 2]$ chips as well as its subsequent $[A / 2]$ chips. Thus, a complete evaluation of the maximum value will involve all different combinations of these continuous $A$ chips. According to Fig. 11, the max occurs when the signs of $I_{n}\left(\right.$ or $Q_{n}$ ) are the same as that of the side lobes of the impulse response for corresponding $n$. The signs of the sidelops are defined as +-++-+ . It is easy to verify that for a given $g_{k}$, the maximum values of the signal envelopes of two spreading schemes are the same but with different probabilities, which are $2 / 8^{A}$ and $2 / 4^{A}$ for complex spreading and dual-channel spreading, respectively. Therefore, the occurrence of peak value is much more frequent for dual-channel spreading than for complex spreading. That is, when a nonlinear power amplifier is used, the nonlinear distortion caused by complex spreading is much less than by dual-channel spreading. In order to quantify 


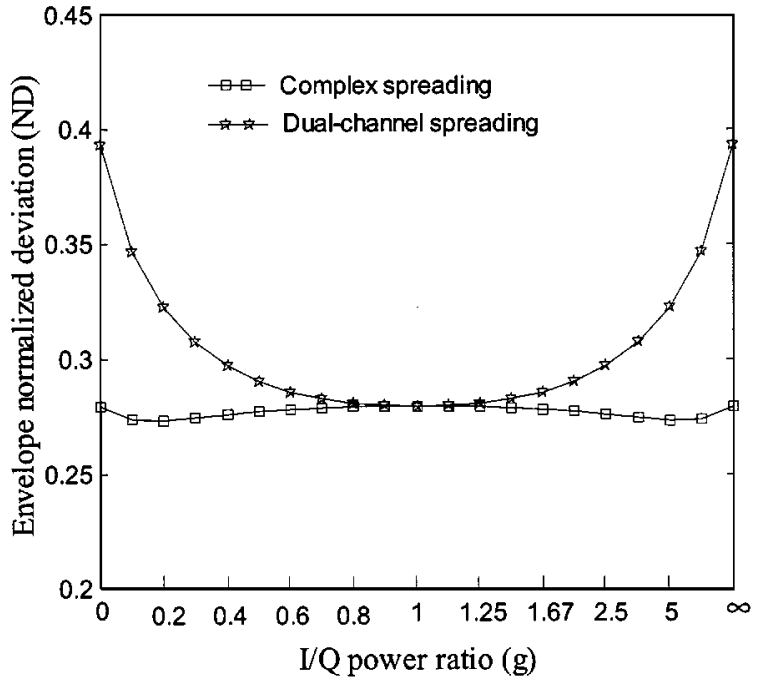

Fig. 12. Envelope deviation of complex and dual-channel spread signals.

this property, the variances and NDs of two spreading signals are evaluated by calculation, which are shown in Table II

It can be observed that the theoretical PARs are the same for both complex and dual-channel spread signals, while the NDs are quite different. In Fig. 12, the NDs are plotted versus the factor $g=g_{k}$ for all $k$, for both complex and dual-channel spread signals. It can be seen that when there is not much power difference between in-phase and quadrature branches, i.e., $g \approx$ 1 , the NDs are the same for both schemes. However, when the power ratio $g$ decreases from 1 , the ND of the dual-channel spread signal increases significantly. That means the backoff of RF power amplifier becomes large when $g$ is small and results in less efficiency. The same situation occurs for dual-channel spreading when $g$ increases from 1. On the other hand, the ND of the complex spread signal remains quite constant when the power ratio changes, which means the power efficiency can be kept as constant. This is obviously a benefit for RF power amplifier design.

\section{CONCLUSION}

In this paper, the performance evaluation of the W-CDMA system with complex spreading and imperfect channel estimation by dedicated pilot symbols is considered. The following conclusions have been drawn.

1) In complex spreading, I- and Q-components of the modulated signal are correlated, whereas in dual-channel spreading, they are independent. Therefore, complex spreading keeps the normalized deviation of signal envelope invariant to the power ratio of in-phase and quadrature components, thus improving the efficiency of RF power amplifier.

2) The system performance degrades significantly due to the channel estimation error, which results from the multiple access and multipath interference. However, this estimation error can be effectively suppressed by LPFs with a large number of taps.
3) The I/Q power ratio can be tuned to achieve the best system performance (the maximum capacity). The optimal value of this power ratio is mainly determined by the number of taps of the LPF and the number of active users in the system. The ratio can be chosen in the range of 0.2 to 0.4 (or -7 to $-4 \mathrm{~dB}$ ).

\section{APPENDIX \\ DERIVATION OF THE EFFECT OF TIMING ERROR}

When the impulse response of the shaping filter is limited to $T_{c}$, the effect of the timing error can be derived from (5)

$$
\begin{aligned}
R(\bar{\tau})= & \int_{n T_{c}+\tau_{1, i}}^{(n+1) T_{c}+\tau_{1, i}} h\left(t-n T_{c}-\tau_{k, l}\right) \\
& \cdot h^{*}\left(t-n T_{c}-\tau_{1, i}\right) d t \\
= & \int_{0}^{T_{c}} h(t-\bar{\tau}) h^{*}(t) d t \\
= & \int_{-\infty}^{\infty} h(t-\bar{\tau}) h^{*}(t) d t
\end{aligned}
$$

where $\bar{\tau}=\tau_{k, l}-\tau_{1, i}$. Assuming $H(f)$ is the frequency response of the shaping filter, (A1) can be written as

$$
\begin{aligned}
R(\bar{\tau})= & \int_{-\infty}^{\infty}\left(\int_{-\infty}^{\infty} H\left(f_{1}\right) e^{j 2 \pi f_{1}(t-\bar{\tau})} d f_{1}\right) \\
& \cdot\left(\int_{-\infty}^{\infty} H^{*}\left(f_{2}\right) e^{-j 2 \pi f_{2} t} d f_{2}\right) d t \\
= & \int_{-\infty}^{\infty} \int_{-\infty}^{\infty} H\left(f_{1}\right) H^{*}\left(f_{2}\right) e^{-j 2 \pi f_{1} \bar{\tau}} \\
& \cdot \int_{-\infty}^{\infty} \int_{-\infty}^{\infty} e^{j 2 \pi\left(f_{1}-f_{2}\right) t} d t d f_{1} d f_{2} .
\end{aligned}
$$

Since $\int_{-\infty}^{\infty} e^{j 2 \pi\left(f_{1}-f_{2}\right) t} d t=\delta\left(f_{1}-f_{2}\right),(\mathrm{A} 2)$ can be further written as

$$
\begin{aligned}
R(\bar{\tau}) & =\int_{-\infty}^{\infty} \int_{-\infty}^{\infty} H\left(f_{1}\right) H^{*}\left(f_{2}\right) e^{-j 2 \pi f_{1} \bar{\tau}} \cdot \delta\left(f_{1}-f_{2}\right) d f_{1} d f_{2} \\
& =\int_{-\infty}^{\infty}\left|H\left(f_{1}\right)\right|^{2} e^{-j 2 \pi f_{1} \bar{\tau}} d f_{1} \int_{-\infty}^{\infty} \delta\left(f_{1}-f_{2}\right) d f_{2} .
\end{aligned}
$$

Noting $\int_{-\infty}^{\infty} \delta\left(f_{1}-f_{2}\right) d f_{2}=1$, one obtains

$$
\begin{aligned}
R(\bar{\tau}) & =\int_{-\infty}^{\infty}\left|H\left(f_{1}\right)\right|^{2} e^{-j 2 \pi f_{1} \bar{\tau}} d f_{1} \\
& =\int_{-\infty}^{\infty}|H(f)|^{2} \cos (2 \pi f \bar{\tau}) d f
\end{aligned}
$$

\section{ACKNOWLEDGMENT}

The authors would like to thank Dr. M. Sawahashi and his team members in the Wireless Laboratories, NTT DoCoMo, Japan, for their many helpful discussions. Their thanks are also 
to the five anonymous reviewers for their extremely constructive reviews, which improved the paper significantly.

\section{REFERENCES}

[1] F. Adachi, M. Sawahashi, and H. Suda, "Wideband DS-CDMA for nextgeneration mobile communications systems," IEEE Commun. Mag., vol. 36, pp. 56-69, Sept. 1998.

[2] E. Dahlman, B. Gudmundson, M. Nilsson, and A. Skold, "UMTS/IMT-2000 based on wideband CDMA," IEEE Commun. Mag., vol. 36, pp. 70-80, Sept. 1998.

[3] T. Ojanpera and R. Prasad, "An overview of air interface multiple access for IMT-2000/UMTS,” IEEE Commun. Mag., vol. 36, pp. 82-95, Sept. 1998.

[4] 3G TS 25.213 version 3.3.0, "Spreading and Modulation (FDD),", 3GPP TSG-RAN, 2000-06.

[5] R. L. Peterson, R. E. Ziemer, and D. E. Borth, Introduction to Spread-Spectrum Communications. Englewood Cliffs, NJ: Prentice-Hall, 1995, p. 63.

[6] L. Staphorst, M. Jamil, and L. P. Linde, "Performance evaluation of a QPSK system employing complex spreading sequences in a fading environment," in Proc. IEEE VTS 50th Vehicular Technology Conf., Sept. 1999, pp. 2964-2968.

[7] T. G. Macdonald and M. B. Pursley, "Complex processing in quaternary direct-sequence spread-spectrum receivers," in Proc. IEEE Military Communications Conf., Oct. 1998, pp. 494-498.

[8] T. Eng and L. B. Milstein, "Coherent DS-CDMA performance in Nakagami multipath fading," IEEE Trans. Commun., vol. 43, pp. 1134-1143, Feb./Mar./Apr. 1995.

[9] — , "Partially coherent DS-SS performance in frequency selective multipath fading," IEEE Trans. Commun., vol. 45, pp. 110-118, Jan. 1997.

[10] G. P. Efthymoglou, V. A. Aalo, and H. Helmken, "Performance analysis of coherent DS-CDMA systems in a Nakagami fading channel with arbitrary parameters," IEEE Trans. Veh. Technol., vol. 46, pp. 289-297, May 1997.

[11] F. Ling, "Pilot assisted coherent DS-CDMA reverse-link communications with optimal robust channel estimation," in Proc. IEEE ICASSP, Apr. 1997, pp. 163-166.
[12] K. Higuchi, H. Andoh, K. Okawa, M. Sawahashi, and F. Adachi, "Experimental evaluation of combined effect of coherent Rake combining and SIR-based fast transmit power control for reverse link of DS-CDMA mobile radio," IEEE J. Select. Areas Commun., vol. 18, pp. 1526-1535, Aug. 2000.

[13] S. Abeta, M. Sawahashi, and F. Adachi, "Performance comparison between time-multiplexed pilot channel and parallel pilot channel for coherent RAKE combining in DS-CDMA mobile radio," IEICE Trans. Commun., vol. E81-B, no. 7, pp. 1417-1425, July 1998.

[14] J. Choi, "Multipath CDMA channel estimation by jointly utilizing pilot and traffic channels," Proc. Inst. Elect. Eng. Commun., vol. 146, no. 5 , pp. 312-318, Oct. 1999.

[15] E. K. Al-Hussaini and A. A. M. al-Bassiouni, "Performance of MRC diversity systems for detection of signals with Nakagami fading," IEEE Trans. Commun., vol. COMM-33, pp. 1315-1319, Dec. 1985.

Jiangzhou Wang (M'91-SM'94) photograph and biography not available at the time of publiction.

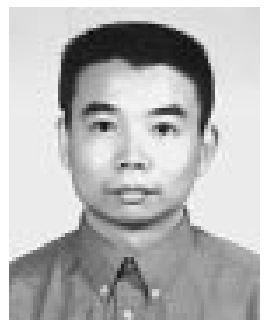

Jun Chen received the B.Eng. degree in electrical engineering and the M.Eng. degree in information and communication engineering from the University of Science and Technology of China, Hefei, in 1996 and 1999 respectively. He is currently pursuing the Ph.D. degree in electrical engineering at the University of Hong Kong, Hong Kong.

From 1995 to 1999, he was with the Personal Communication and Spread Spectrum Laboratory, University of Science and Technology of China, as a Research Engineer involved in the development of prototype CDMA communication systems. His research interests are in the area of mobile communication system, wireless networking, and VLSI implementation of wireless transceivers. 